\title{
Klinische Versuche, Haftpflicht und Versicherungsverträge
}

\author{
D. Sprumont, S. Boillat, H. Amstad
}

1 Dabei sollte man nicht vergessen, dass sich diese Probleme nicht nur in der Schweiz, sondern auch auf internationaler Ebene stellen. Dazu siehe auch: Decoster G, Smith J. Insuring international clinical trials: The killjoy of the harmonization process.

Good Clinical Practice Journal 1998;5(3):13-5.

2 Bundesgesetz über Arzneimittel und Medizinprodukte, 15. Dezember 2000 (Heilmittelgesetz, HMG), SR 812.21.

3 Die AG StaR setzt sich aus Vertreterinnen und Vertretern der Swissmedic, Schweizerischen Akademie der Medizinischen Wissenschaften, des BAG, der Ethikkommissionen und Kantonsapotheker zusammen. Sie wurde als Forum für Diskussionen und Reflexionen zu Fragen der Umsetzung der Reglementierung der klinischen Versuche und insbesondere der Praxis der EKF geschaffen.

4 Verordnung über klinische Versuche mit Heilmitteln (Vklin) vom 17. Oktober 2001, SR 812.214.2

5 Sprumont D, Béguin M-L. La nouvelle réglementation des essais cliniques de médicaments. Bull Méd Suisses 2002;83 (18):894-906. Sprumont D. La protection des sujets de recherche, notamment dans le domaine biomédical. Stämpfli, Berne 1993, p. 234 ss (et auteurs cités).

Korrespondenz:

Prof. Dominique Sprumont Institut de droit de la santé Université de Neuchâtel 26, Avenue du $1^{\text {er }}$ Mars 26 CH-2000 Neuchâtel
Seit kurzem beschäftigen sich sowohl die Pharmaindustrie als auch Ethikkommissionen für Forschungsuntersuchungen am Menschen (EK) und Behörden mit Fragen der Haftpflicht bzw. der Versicherungspflicht von Prüfern und Sponsoren. Die Pharmaindustrie beklagt sich darüber, dass die EK in der Beurteilung der den Forschungsprojekten beigelegten Versicherungsausweise nicht kohärent seien; die EK ihrerseits können nur feststellen, dass die Situation einer Klärung bedarf. Sollte in dieser Frage keine gemeinsame Lösung gefunden werden, sehen manche das Weiterbestehen des Forschungsplatzes Schweiz gefährdet, denn die Pharmaindustrie werde es vorziehen, ihre Studien in Ländern mit klareren Regeln [1] durchzuführen. Selbst das Inkrafttreten des neuen Heilmittelgesetzes (HMG) [2] konnte die Gemüter nicht beruhigen.

Ausgehend von dieser eher kritischen Bilanz wurde die Arbeitsgruppe «Standardisierung und Registrierung» (AG StaR) [3] von der Schweizerischen Akademie der Medizinischen Wissenschaften (SAMW), von Vertretern der EK und der eidgenössischen und kantonalen Gesundheitsbehörden aufgefordert, die konkreten Probleme in diesem Bereich zu analysieren und Empfehlungen zuhanden der EK und der Behörden abzugeben. Obwohl die AG StaR ihre Arbeit noch weiterführt, halten es die Autoren/-innen dieses Artikels für nützlich, erste Schlussfolgerungen $\mathrm{zu}$ veröffentlichen. Damit soll eine unnötige Zuspitzung der Lage verhindert und eine rasche Suche nach Lösungen erleichtert werden. Es geht in erster Linie darum, in einem Überblick die praktischen Probleme darzustellen, mit denen die EK konfrontiert werden, und ihnen eine Art Checkliste vorzuschlagen, die bei der Beurteilung von Forschungsprojekten hilfreich sein könnte.

Das Thema Haftpflicht und Versicherungsschutz im Bereich der klinischen Versuche wird von drei verschiedenen Seiten her angegangen: 1. eine rechtliche Analyse der Sachlage mit einer kurzen rechtsvergleichenden Darstellung; 2. eine retrospektive Studie der Versicherungsausweise, die den von der Überregionalen Ethikkommission (UREK) der SAMW zwischen 1997 und 2000 evaluierten Forschungsprojekten beilagen; und
3. der Versand eines Fragebogens an alle EK, in dem sie über ihre jeweilige Praxis in dieser Sache befragt wurden. Wir werden unsere Ergebnisse in der gleichen Reihenfolge präsentieren.

\section{Haftpflicht und Versicherungsschutz in der Gesetzgebung über klinische Versuche mit Heilmitteln}

Das Heilmittelgesetz (HMG) und die Verordnung über klinische Versuche mit Heilmitteln (VKlin) [4], die am 1. Januar 2002 in Kraft getreten sind, legen keine präzisen Regeln für die Haftpflicht von Sponsoren und Prüfern fest. Einige Bestimmungen beziehen sich jedoch auf diesen Problembereich. Artikel 54 Buchstabe b HMG legt fest: «Es ist gewährleistet, dass die Versuchspersonen für allfällige Schäden, die sie im Rahmen eines Versuchs erlitten haben, vollumfänglich entschädigt werden.» Die VKlin präzisiert in Artikel 7, dass der Sponsor der Versuchsperson den Schaden ersetzt, den sie im Rahmen eines klinischen Versuchs erleidet, und dass er diese Verpflichtung sicherstellen muss. Er kann allerdings Rückgriff nehmen auf die Prüferin oder den Prüfer oder auf andere Personen, die ebenfalls gegenüber der Versuchsperson für den Schaden haften. Hat der Sponsor seinen Sitz im Ausland, so muss er eine Person in der Schweiz bezeichnen, welche die Verpflichtung sicherstellt. Der Versuchsperson muss zudem ein direkter Anspruch gegen diese Person eingeräumt werden.

Im Gegensatz zur Beziehung Patient-Arzt ist es hier die Versuchsperson, die dem Prüfer und dem Sponsor eine Dienstleistung erbringt (Beziehung Versuchsperson-Prüfer bzw. Sponsor). Damit wird der Haftungsgrad für Prüfer und Sponsor erhöht. Diese Verschärfung der Haftpflicht betrifft einerseits die Tatsache, dass sie sogar dann gilt, wenn auf seiten des Schadenverursachers kein Fehlverhalten vorliegt; ausserdem ist die Beweislast für die Versuchsperson, dass zwischen dem erlittenen Schaden und dem Versuch ein entsprechender Kausalzusammenhang besteht, vermindert [5]. Artikel $54 \mathrm{HMG}$ spricht von «der Deckung der Schäden, die sie im Rahmen eines Versuchs erlitten haben». Somit 
Tabelle 1

Die Situation in den Nachbarstaaten [8].

\begin{tabular}{|c|c|c|c|}
\hline Fragestellung & Deutschland & Frankreich & Österreich \\
\hline obligatorischer Versicherungsschutz & ja für Produkte nach AMG und MPG & ja & ja für Produkte nach AMG und MPG \\
\hline Poollösung & $\begin{array}{l}\text { Produkte nach AMG werden } \\
\text { über den Pharmapool versichert } \\
\text { (Rückversicherer: Münchner Rück) }\end{array}$ & nein & nein \\
\hline \multicolumn{4}{|l|}{ Versicherungssumme (in $€$ ) } \\
\hline je Proband & 500000 & 760000 & 370000 \\
\hline - bis 1000 Probanden & 10000000 & & \\
\hline - bis 3000 Probanden & 20000000 & & \\
\hline - über 3000 Probanden & 30000000 & & \\
\hline je Studie & & 4570000 & 2500000 \\
\hline Nachversicherung & $\begin{array}{l}\text { Zwei Jahre nach Ende des Jahres, } \\
\text { in welchem die Studie beendet wurde }\end{array}$ & $\begin{array}{l}10 \text { Jahre nach Ablauf } \\
\text { des Versuchs }\end{array}$ & 3 Jahre nach Ablauf des Versuchs \\
\hline Bemerkungen & & $\begin{array}{l}\text { Versicherungsschutz } \\
\text { zurzeit schwer erhältlich }\end{array}$ & \\
\hline
\end{tabular}

6 Bundesgesetz vom 30. März 1911 betreffend die Ergänzung des Schweizerischen Zivilgesetzbuches (Fünfter Teil: Obligationenrecht), SR 220.

7 Für eine rechtsvergleichende Darstellung siehe: Decoster G, Wahl M. Insuring subjects in Clinical Trials. Applied Clin Trials 1998;7:30-6.

8 Die Autoren danken Ruedi Dösseger von True Partners AG, Insurance Brokers, Rapperswil, für das Zurverfügungstellen dieser Tabelle.

9 Für eine Analyse aller Aktivitäten dieser Kommission vgl. Truniger B, Spahr A, Pletscher W, Amstad H. Ethikkommissionen zwischen Anspruch und Wirklichkeit Rückblick auf neun Jahre «Überregionale Ethikkommission» (UREK) der SAMW. Schweiz Ärztezeitung 2001;82(45):2398-403. genügt es, wenn die Versuchsperson aufzeigen kann, dass der Schaden, den sie erlitten hat, aller Wahrscheinlichkeit nach in Zusammenhang mit dem Versuch steht. Es obliegt dann dem Sponsor, gegebenenfalls darzulegen, dass ein solcher $\mathrm{Zu}$ sammenhang nicht besteht. Die von HMG und VKlin vorgeschlagene Lösung soll vor allem die Lage einer geschädigten Versuchsperson vereinfachen. Sie stellt sicher, dass die Versuchsperson sich für die Entschädigung nur an einen einzigen Verantwortlichen wenden muss. Der Ausdruck «vollumfängliche» Entschädigung bezieht sich auf die Gesamtheit der Schäden, wie dies im Obligationenrecht [6] vorgesehen ist. Ein Sponsor kann also seine Haftpflicht nicht nur auf körperliche Schäden beschränken, wie dies manchmal der Fall zu sein scheint.

Auch wenn die VKlin den Sponsor als Hauptverantwortlichen bezeichnet, so geht sie doch nicht so weit, ihm eine Versicherungspflicht vorzuschreiben. Es geht allein um die Möglichkeit, eine Versicherung abzuschliessen. Nur die EK und gegebenenfalls Swissmedic können verlangen, dass ein Sponsor eine Haftpflichtversicherung abschliesst, wenn die von ihm geleisteten Garantien als ungenügend erscheinen, um die Interessen der Versuchspersonen sicherzustellen. In der Regel haben jedoch nur wenige Sponsoren keine Haftpflichtversicherung. In der Praxis unterscheidet sich die Situation in der Schweiz nicht so sehr von derjenigen der Nachbarländer [7] (vgl. Tab. 1). Es wäre jedoch wünschenswert, wenn das zukünftige Gesetz über die Forschung am Menschen in seinen Ausführungsbestimmungen die Versicherungspflicht einführen würde. Dies würde die Aufgabe der EK erleichtern und die rechtsgleiche Behandlung aller Beteiligten gewährleisten.

\section{Analyse der verschiedenen Versicherungsausweise: Wichtigste Problembereiche}

Zwischen 1997 und 2000 wurden bei der UREK der Schweizerischen Akademie der Medizinischen Wissenschaften 52 Forschungsprojekte zur Begutachtung eingereicht [9]. Die Analyse der verschiedenen Projekte unter dem Gesichtspunkt Haftpflicht und Versicherungsschutz zeigte mehrere kritische Punkte auf, die einer Klärung bedürfen. Diese Analyse wurde durch eine Umfrage bei den EK ergänzt, um deren Evaluationskriterien in diesem Bereich in Erfahrung zu bringen. Tatsächlich ist es - neben der differenzierten Beurteilung jedes einzelnen Forschungsprojekts durch die EK - die grosse Vielfalt von Versicherungsverträgen, die mit den Projekten eingereicht werden, welche die Aufgabe der EK nicht eben leicht macht. Es geht nun darum, besser zu verstehen, wo die Hauptprobleme im Bereich Haftpflicht und Versicherungsschutz im Rahmen der biomedizinischen Forschung in der Schweiz liegen.

Auch wenn die Anzahl der analysierten Dossiers klein ist, können doch gewisse allgemeine Tendenzen für die Gesamtheit der Versicherungsverträge ausgemacht werden, die während der betreffenden Periode mit Forschungsprojekten in der Schweiz in Zusammenhang stehen. Bei der 
Mehrzahl der Projekte handelte es sich um Multizenterstudien. Obwohl die Aktivitäten der UREK aus quantitativer Sicht nicht sehr bedeutend waren, musste sie doch einige komplexe Projekte beurteilen. Die Tatsache, dass die UREK den hier erwähnten Problemen mit Versicherungsverträgen begegnet ist, lässt vermuten, dass es den andern EK in unserem Land in dieser Hinsicht auch nicht besser gegangen ist.

\section{Fehlender Versicherungsausweis}

Über ein Drittel der analysierten Forschungsprojekte machten in keiner Weise Bezug auf irgendeinen Versicherungsausweis. Bei bestimmten Forschungsarten - z. B. bei epidemiologischen Studien - ist dies leicht zu verstehen. Doch bei klinischen Versuchen sollte es die Ausnahme bleiben, auch wenn das Gesetz es zulässt. Es ist wichtig, dass die EK bei fehlendem Versicherungsvertrag genau prüft, ob die Interessen der Versuchspersonen tatsächlich gewahrt werden. Im Zweifelsfall hat die EK die Kompetenz, die Garantie zu verlangen, die durch einen Versicherungsvertrag gegeben ist. Es geht jedoch nicht darum, dass die EK die Bilanz eines Unternehmens anfordern oder zum Finanzanalysten werden muss. Es liegt in der Verantwortung des Sponsors nachzuweisen, dass er die mit der Studie verbundenen Risiken decken kann; er allein ist den Versuchspersonen gegenüber verantwortlich.

\section{Vielzahl von unterschiedlichen Verträgen, anwendbares Recht, Gerichtsstand}

Bezogen auf die Gesamtheit der evaluierten Verträge sind es vier Versicherungsgesellschaften, die Standardverträge mit identischen Bestimmungen - unabhängig vom versicherten Forschungsprojekt - vorschlagen. Dazu kommen weitere vier Gesellschaften, welche die Risiken von mehr als einer Studie abdecken. Es gibt also kein einheitliches Vertragsmodell der 17 Versicherungsgesellschaften, welche die verschiedenen analysierten Verträge unterschrieben haben. Im Gegenteil, es muss noch hervorgehoben werden, dass einige dieser Versicherungsgesellschaften eine Adresse im Ausland haben, z. B. in Deutschland, Frankreich und sogar auf den Bermudas, ohne Angabe des anwendbaren Rechts für die jeweiligen Verträge.

Wenn man sich bewusst ist, dass in manchen Fällen der Sponsor einer Studie eine ausländische Firma ist, versteht man die Probleme im Zusammenhang mit dem anwendbaren Recht noch besser. Die Forderung in Artikel 7 der VKlin, dass eine Person mit Schweizer Wohnsitz die Verpflichtungen des Sponsors sicherstellen muss, ist daher mehr als berechtigt. Abgesehen von der
Notwendigkeit, das anwendbare Recht zu präzisieren, muss auch gefordert werden, dass in einem Streitfall der Gerichtsstand derjenige der geschädigten Versuchsperson ist. Des weitern muss gewährleistet werden, dass ihr ein direkter Anspruch gegenüber der Versicherung eingeräumt wird. Die Sicherstellung eines solchen direkten Anspruchs scheint angemessen, weil so den Versuchspersonen langwierige Rechtshändel zur Geltendmachung ihrer Rechte beim Sponsor erspart werden können.

\section{Versicherungsnehmer und versicherte Personen}

Auf den Informationsblättern bestehen oft Unklarheiten über die Frage, wer effektiv versichert ist. Gemäss den Vorschriften über Haftpflichtversicherungsverträge kann dies nicht die Versuchsperson sein, sondern einzig und allein der Sponsor oder - eher selten zwar - der Prüfer oder die Prüferin. Das Resultat unserer Untersuchung zeigt, dass 5 Verträge, davon ein Standardvertrag, angeben, der Sponsor (bzw. das Pharmaunternehmen) sei die versicherte Person, während die Prüfenden als der «Certificate Holder» oder Versicherungsnehmende bezeichnet werden. In allen andern Fällen werden nur die Pharmaunternehmen als versicherte Person aufgeführt.

In diesem Zusammenhang ist es wichtig zu prüfen, ob der Kreis der durch den Vertrag gedeckten Personen so gross wie möglich gezogen wird und nicht nur den Sponsor und den Prüfer, sondern auch deren Mitarbeitende und alle andern am Versuch beteiligten Personen umfasst. Ebenso wichtig ist es zu ermitteln, ob im Schadenfall andere Haftpflichtversicherungen mit einbezogen werden können, beispielsweise wenn die Studie in einem Spital durchgeführt wird, das über eine eigene Haftpflichtversicherung für Forschungsprojekte verfügt. In diesem Fall muss von den Prüfern verlangt werden, dass sie die Sachlage vor Beginn der Studie klären, denn sonst könnte sich die geschädigte Versuchsperson in einen Zuständigkeitsstreit unter den Versicherungen verwickelt sehen, der zu ihren Ungunsten ausgehen könnte.

\section{Versicherungsart}

27 Verträge geben die Haftpflichtversicherung als Versicherungsart an. Die meisten tun dies in einem umfassenden Sinn (Versicherung für klinische Versuche). In einem Fall handelt es sich allerdings nur um eine Produktehaftpflicht [10], was nicht den gesetzlichen Anforderungen entspricht. Es ist also wichtig, die Versicherungsart zu kontrollieren und zu verlangen, dass es sich effektiv um eine Haftpflichtversicherung handelt. 


\section{Art der versicherten Schäden}

Die versicherten Schäden sind meistens nicht definiert. Nur 14 der 34 Verträge sagen etwas dazu aus. Die meisten sehen explizit die Übernahme von körperlichen Schäden und Sachschaden vor, 5 Verträge beziehen sich hingegen nur auf körperliche oder gesundheitliche Schäden. In einem Fall wird erwähnt, dass «genetische Schäden» nicht gedeckt sind. Glücklicherweise sind solche Einschränkungen heute mehr und mehr am Verschwinden. Die Tatsache, dass die Haftpflichtversicherung gewisse Schäden ausschliesst, darf allerdings nicht als eine Einschränkung der Verantwortung des Sponsors gedeutet werden. In diesem Zusammenhang muss sichergestellt werden, dass das Informationsblatt keine Bestimmung enthält, die bei den Versuchspersonen den Gedanken aufkommen lassen könnte, dass sie nur für bestimmte Schadensarten eine Entschädigung verlangen dürfen. Ein Verweis auf Artikel 54 HMG auf dem Informationsblatt würde klarstellen, dass alle versuchsbedingten Schäden vollumfänglich entschädigt werden.

\section{Ausmass der Schadendeckung}

Die Versicherungssummen variieren stark (zwischen Fr. 1000 000.- und DM 100000 000.-). Einige Verträge erwähnen einzig den Gesamtbetrag, andere präzisieren die Versicherungssummen pro Forschungsprojekt und Versuchsperson. Manchmal wird ein Höchstbetrag von $€ 500$ 000.- [11] und Fr. 1000 000.- pro Versuchsperson festgelegt; dabei unterscheiden gewisse Verträge zwischen den Versicherungssummen für gesundheitliche Schäden und denen für Sachschäden. Schliesslich muss erwähnt werden, dass die meisten Verträge eine Franchise von Fr. 5000.- bis 50000 .- beinhalten. In den Dossiers ist jedoch nicht ersichtlich, wer diese Franchise übernehmen sollte. Es darf sich aber auf keinen Fall um die Versuchsperson handeln. Unabhängig von den unterschiedlichen Anforderungen der EK ist festzustellen, dass es im Bereich der Versicherungsverträge kaum eine Koordination gibt. Zu den Elementen, die diese Sachlage noch erschweren, gehört, dass die jeweiligen Versicherungssummen sowohl in Schweizer Franken als auch in DM, US-Dollars oder schwedischen Kronen angegeben werden. Auch wenn der Euro die Sache vereinfachen sollte, fehlt eine einheitliche Lösung.

Vom Standpunkt der EK aus gesehen ist das Ausmass der Schadendeckung besonders wichtig. Sie fragen sich oft, ob die angegebene Versicherungssumme ausreichend ist. Diese Frage muss allerdings in einem Gesamtzusammenhang betrachtet werden. Denken wir beispiels- weise daran, dass die Versicherungssumme von Fr. 3000 000.- für die Autohaftpflichtversicherung den Gesamtschaden je Unfallereignis für Personen- und Sachschaden zusammen deckt [12]. Im Vergleich dazu scheint auf den ersten Blick ein Betrag von Fr. 500 000.- angemessen für den gesamten Schaden, den eine Versuchsperson erleiden kann. Diese Summe entspricht beispielsweise dem Minimalbetrag, den die in der Schweiz ansässigen Luftfahrtgesellschaften pro Passagier garantieren müssen, entweder durch Eigenfinanzierung oder mittels einer Haftpflichtversicherung bei einer Versicherungsgesellschaft, die in der Schweiz zugelassen ist [13]. Nach Artikel 22 des Warschauer Abkommens vom 12. Oktober 1929 wird diese Summe halbiert (Fr. 250 000.-), wenn es um die Haftpflicht der andern Luftfahrtgesellschaften geht [14].

In diesem Fall muss man eine gewisse Flexibilität zeigen; spezifische Forderungen können jedoch je nach Umständen entsprechend gerechtfertigt sein. Wenn beispielsweise ein klinischer Versuch ein hohes Risiko beinhaltet und die Versuchsperson erwerbstätig und für den Unterhalt einer Familie verantwortlich ist, muss das effektive Ausmass eines allfälligen Schadens abgeschätzt werden. Gegebenenfalls ist die EK berechtigt, eine entsprechende Erhöhung der Schadendeckung zu verlangen.

\section{Versicherungsdauer}

In 16 Verträgen stimmt die Versicherungsdauer nicht mit der Gesamtdauer des klinischen Versuchs überein. Es werden keine Angaben darüber gemacht, wie Schäden im Rahmen des Versuchs gedeckt werden, die vor oder nach der im Vertrag festgelegten Versicherungsdauer entstehen. Für die Versuchsperson ist es ausserdem wichtig zu wissen, bis wann die Haftpflicht gilt, falls sie nachträglich einen Schaden feststellen sollte. Diese Frage wird in den analysierten Dossiers nicht behandelt.

Es sollte daher in jedem Fall überprüft werden, ob die Versicherungsdauer effektiv mit der Dauer des Versuchs übereinstimmt. Zudem müssen genaue Angaben darüber verlangt werden, bis wann eine geschädigte Versuchsperson eine Entschädigung einfordern kann. Die gesetzliche Frist für die Haftpflicht beträgt 10 Jahre, doch es kommt häufig vor, dass die Versicherungsverträge nicht länger als fünf oder sogar nur zwei Jahre gültig sind. Diese Information muss den Versuchspersonen unbedingt gegeben werden, denn ein einfacher Verweis auf die gesetzliche Verjährungsfrist ist unzureichend, weil die Versicherungsdauer nicht mit ihr übereinstimmt. 


\section{Fragebogen an die Präsidenten der EK betreffend Haftpflicht und Haftpflichtversicherung}

Die letzte Bemerkung führt uns direkt zu der dritten Phase unserer Analyse. Dabei ging es darum, mehr darüber zu erfahren, wie die EK selbst die Situation einschätzen.

Erste Feststellung: von den 1598 Projekten, die 2001 von den EK beurteilt wurden, gab es nur bei 193 Probleme im Zusammenhang mit dem Versicherungsvertrag. Dies könnte den Eindruck erwecken, das Problem sei nicht so bedeutend, wie manche behaupten, dies um so mehr, als etliche dieser Projekte Multizenterstudien sind. Da es sich dabei aber oft um Versuche mit Medikamenten handelt und diese in der Schweiz fast 500 pro Jahr ausmachen, ist offensichtlich, dass das Problem heikler ist, als es auf den ersten Blick erscheinen mag.

Die wichtigsten Gründe, welche die EK veranlasst haben, zusätzliche Informationen zu verlangen, sind in Tabelle 2 wiedergegeben.

Tabelle 2

Wichtigste Problembereiche gemäss EK.

\begin{tabular}{ll} 
Fehlender Versicherungsvertrag & 33 \\
\hline Zweifelhafter Versicherungsausweis & 61 \\
\hline Ungenügende Schadendeckung & 35 \\
\hline Ungenügende Versicherungsdauer & 47 \\
\hline Andere & 17
\end{tabular}

Während die Frage nach der Schadendeckung vor allem die Pharmaindustrie interessiert, stellt man fest, dass nur 7 EK dazu spezifische Forderungen stellen, die von Fr. 1000 000.- bis 3000 000.- pro geschädigte Versuchsperson und Fr. 5000 000.- bis 10000 000.-pro Studie reichen. Wie oben bereits gesagt, sollte man in dieser Sache keine allzu starre Haltung einnehmen, in der Hoffnung, dass diese Frage im Gesetz geregelt wird.

\section{Schlussfolgerung}

Alles in allem zeigt unsere Analyse, dass es im Bereich der klinischen Versuche mit Heilmitteln im Zusammenhang mit der Haftpflichtversicherung viele Probleme gibt. Der Problemkatalog ist nicht abschliessend. So fehlen fast systematisch die allgemeinen Versicherungsbedingungen, die für eine Analyse des Vertrags unerlässlich sind. Der Problemkatalog, den wir hier erstellt haben, sollte als Referenz für die Evaluation von Forschungsprojekten durch die EK dienen. Es ist wichtig, dass sie jeden einzelnen dieser Punkte überprüfen, um zu entscheiden, ob das vorgeschlagene Verfahren ausreichend ist. Das heisst insbesondere, dass sie sich nicht einfach mit einem Versicherungsausweis begnügen dürfen, sondern dass sie den Vertrag selbst und vor allem seine allgemeinen Bedingungen zur Einsicht verlangen müssen.

Man kann sich im übrigen nur über die grossen Unterschiede zwischen diesen Versicherungsverträgen und dem Mangel an Kohärenz wundern. Das Recht der Versuchspersonen auf eine Entschädigung im Falle eines Schadens leidet unter der fehlenden Aufmerksamkeit von Sponsoren und Prüfern [15]. Es wäre deshalb sehr nützlich, wenn die Pharmaindustrie ihre eigenen Richtlinien aufstellen würde, um die Situation zu verbessern und gegebenenfalls Musterverträge vorschlagen würde, welche $a$ priori die gesetzlichen und ethischen Forderungen respektieren. Solche Musterverträge existieren. Viele der heutigen Probleme könnten bereits gelöst werden, wenn die Dachverbände der Pharmaindustrie (z. B. Interpharma und die Schweizerische Gesellschaft für Chemische Industrie) nur schon ihre Mitglieder auffordern würden, diese Musterverträge zu verwenden. Indem sie ihre Verantwortung gegenüber den Versuchspersonen und der Gesellschaft wahrnimmt, würde die Pharmaindustrie zeigen, dass sie tatsächlich gewillt ist, eine Lösung zu finden.

Eine weitere Alternative wäre natürlich, eine Versicherungspflicht gesetzlich oder behördlich festzulegen. Doch dies würde mit einem grossen Zeitaufwand verbunden sein, und über diese Zeit verfügt die Industrie - wie sie richtigerweise klagt - nicht.

Abschliessend muss das Vorgehen der EK begrüsst werden, die sich nicht einfach vor vollendete Tatsachen stellen lassen und sie Projekte akzeptieren, die im Bereich der Sponsorenhaftpflicht und der Haftpflichtversicherung lückenhaft sind. Wir sind uns allerdings bewusst, dass es hier um eine sehr komplexe Frage geht, und empfehlen den EK, sich im Zweifelsfall an Swissmedic zu wenden. Im übrigen bereitet Swissmedic unseres Wissens Empfehlungen zum Thema Haftpflichtversicherung vor, die hier Klarheit schaffen sollen.
The right(s) of the subject in clinical trial-related injury. Good Clinical Practice Journal 1998;5( 6):11-5. 